\title{
Fermentative Spirochaetes mediate necromass recycling in anoxic hydrocarbon-contaminated habitats
}

\author{
Xiyang Dong ${ }^{1,2} \cdot$ Chris Greening $^{3} \cdot$ Thomas Brüls $^{4,5} \cdot$ Ralf Conrad $^{6} \cdot$ Kun Guo $^{7}$ - Svenja Blaskowski ${ }^{8}$. \\ Farnusch Kaschani $^{8} \cdot$ Markus Kaiser $^{8} \cdot$ Nidal Abu Laban $^{1} \cdot$ Rainer U. Meckenstock $^{1}$
}

Received: 28 October 2017 / Revised: 23 March 2018 / Accepted: 29 March 2018 / Published online: 30 May 2018

(c) The Author(s) 2018. This article is published with open access

\begin{abstract}
Spirochaetes are frequently detected in anoxic hydrocarbon- and organohalide-polluted groundwater, but their role in such ecosystems has remained unclear. To address this, we studied a sulfate-reducing, naphthalene-degrading enrichment culture, mainly comprising the sulfate reducer Desulfobacterium N47 and the rod-shaped Spirochete Rectinema cohabitans HM. Genome sequencing and proteome analysis suggested that the Spirochete is an obligate fermenter that catabolizes proteins and carbohydrates, resulting in acetate, ethanol, and molecular hydrogen $\left(\mathrm{H}_{2}\right)$ production. Physiological experiments inferred that hydrogen is an important link between the two bacteria in the enrichment culture, with $\mathrm{H}_{2}$ derived from fermentation by $R$. cohabitans used as reductant for sulfate reduction by Desulfobacterium N47. Differential proteomics and physiological experiments showed that $R$. cohabitans utilizes biomass (proteins and carbohydrates) released from dead cells of Desulfobacterium N47. Further comparative and community genome analyses indicated that other Rectinema phylotypes are widespread in contaminated environments and may perform a hydrogenogenic fermentative lifestyle similar to $R$. cohabitans. Together, these findings indicate that environmental Spirochaetes scavenge detrital biomass and in turn drive necromass recycling at anoxic hydrocarbon-contaminated sites and potentially other habitats.
\end{abstract}

Electronic supplementary material The online version of this article (https://doi.org/10.1038/s41396-018-0148-3) contains supplementary material, which is available to authorized users.

Rainer U. Meckenstock

rainer.meckenstock@uni-due.de

1 University of Duisburg-Essen, Biofilm Centre, Universitätsstrasse 5, 45141 Essen, Germany

2 Helmholtz Zentrum München, Institute of Groundwater Ecology, Ingolstädter Landstrasse 1, 85764 Neuherberg, Germany

3 Monash University, Centre for Geometric Biology, School of Biological Sciences, Clayton, VIC 3800, Australia

4 CEA, DRF, IG, Genoscope, Evry 91057, France

5 CNRS-UMR8030 and Université Paris-Saclay, UEVE, Evry 91000, France

6 Department of Biogeochemistry, Max Planck Institute for Terrestrial Microbiology, Karl-von-Frisch-Strasse 10, D-35043 Marburg, Germany

7 Ghent University, Center for Microbial Ecology and Technology, Coupure Links 653, B-9000 Ghent, Belgium

8 University of Duisburg-Essen, Centre of Medical Biotechnology, Universitätsstrasse 2, 45117 Essen, Germany

\section{Introduction}

The bacterial phylum Spirochaetes is well known for its commensal and pathogenic species, among them the causative agents of syphilis and Lyme disease [1]. However, 16S rRNA gene sequences of environmental Spirochaetes are also frequently detected in anoxic environments, including sites contaminated with organic pollutants [2, 3]. Environmental Spirochaetes are also present in many enrichment cultures that degrade hydrocarbons or chlorinated compounds under anoxic conditions, including toluene [4], naphthalene [5], alkanes [2], terephthalate [6], and trichloroethene [7, 8]. For example, the relative abundances of Spirochaetes range from 1.4 to $20.8 \%$ in 10 reported sulfate-reducing or methanogenic hydrocarbondegrading enrichment cultures originating from hydrocarbon-contaminated groundwater, aquifer sediments, and oil sands tailings ponds [2,9]. In these cultures, sulfatereducing or fermenting bacteria primarily mediate the anaerobic transformation of hydrocarbon compounds [10]. In contrast, the functional role of the Spirochaetes has remained unresolved. 
The recent isolation of Sphaerochaeta pleomorpha and Sphaerochaeta globosa from trichloroethene-dechlorinating enrichment cultures provided evidence that some environmental Spirochaetes are morphologically and metabolically distinct from host-associated representatives from the phylum [8]. Contrary to other Spirochaetes, which are motile and spiral-shaped, the two strains are rod-shaped, nonmotile microorganisms that lack flagella [7, 8]. It was proposed that these environmental Spirochaetes might serve as autotrophic acetogens $[11,12]$, but homoacetogenic growth with $\mathrm{H}_{2}-\mathrm{CO}_{2}$ was not observed [8]. Genome-based inferences of the metabolic capacity of these isolates suggested a fermentative and saccharolytic lifestyle [7]. Nevertheless, their functional role was untested experimentally and remains unclear.

We have shown that, under sulfate-reducing conditions, naphthalene can be anaerobically mineralized to $\mathrm{CO}_{2}$ by the stable enrichment culture N47 derived from sediments of a contaminated aquifer [5, 13]. Based on 16S rRNA gene sequence analysis, $\sim 93 \%$ of the culture comprised a hitherto uncultured sulfate reducer Desulfobacterium strain N47 that has been shown to be responsible for naphthalene degradation [5, 14]. The remainder of the enrichment culture mainly comprised a Spirochete, Rectinema cohabitans strain HM, which is neither able to degrade naphthalene nor reduce sulfate or other sulfur compounds in pure culture [15]. Other possible species might exist in this enrichment with unknown functions, but were not detected by terminal restriction fragment length polymorphism fingerprinting [15] because they represented less than $1 \%$ of the total DNA [16].

Here, we intended to elucidate the functional role of environmental Spirochaetes using the enrichment culture $\mathrm{N} 47$ as an example. By means of physiological, proteogenomic, and comparative genome analyses, we show that environmental Spirochaetes are involved in necromass recycling and provide hydrogen and nutrients to associated hydrocarbon degraders. Through reconstructing the genomes of two other putative Rectinema species, we also provide evidence that environmental Spirochaetes may generally contribute to necromass recycling in anoxic hydrocarbon- and organohalide-contaminated environments as well as other anoxic habitats.

\section{Materials and methods}

\section{Bacterial cultures and cultivation}

The naphthalene-degrading, sulfate-reducing enrichment culture N47 was cultivated in bicarbonate-buffered freshwater medium as described previously [5]. After autoclaving, $3 \mathrm{~mL}$ of $1.5 \%(\mathrm{w} / \mathrm{v})$ naphthalene dissolved in $2,2,4,4,6,8,8$ heptamethylnonane (Sigma-Aldrich, Steinheim, Germany) were added to each cultivation bottle. Enrichment culture N47 mainly contained a sulfate reducer, candidate Desulfobacterium strain N47, and a spirochete, Rectinema cohabitans [15].

Attempts to isolate Desulfobacterium strain N47 in pure culture by serial dilution in agar roll tubes resulted in a highly enriched Desulfobacterium culture that lacked the Spirochete R. cohabitans and was $96 \%$ dominated by Desulfobacterium strain N47 (Figure S2, details in Supporting Information). The culture performed sulfate reduction and naphthalene oxidation and was cultivated in the same way as the original enrichment culture. This culture is described as "highly enriched Desulfobacterium culture" hereafter. R. cohabitans strain HM [15] was isolated from the enrichment culture N47 and cultivated in bicarbonate-buffered freshwater medium $(\mathrm{pH} 7.0)$, which was reduced with a final concentration of $0.5 \mathrm{mM}$ sodium sulfide and amended with $10 \mathrm{mM}$ glucose and $0.1 \%$ yeast extract. All culture bottles were incubated stationary at $30^{\circ} \mathrm{C}$ in the dark.

To test whether $R$. cohabitans can utilize dead biomass of Desulfobacterium N47 as a carbon source, cells of the highly enriched Desulfobacterium culture were harvested from $150 \mathrm{~mL}$ cultures by centrifugation at $3214 \times g$ (30 min). The supernatant, together with the heptamethylnonane phase containing the naphthalene, was discarded. The cell pellets were suspended in $2.5 \mathrm{~mL}$ anoxic fresh medium (without substrate or yeast extract), and autoclaved three times in a closed $100 \mathrm{~mL}$ serum bottle at $121^{\circ} \mathrm{C}$ for $20 \mathrm{~min}$. Thereafter $0.5 \mathrm{~mL}$ dead biomass was transferred to serum bottles filled with $30 \mathrm{~mL}$ fresh medium and the bottles were inoculated with $3 \mathrm{~mL}$ cultures of $R$. cohabitans. Due to the carryover from the previous cultures, glucose, yeast extract, and fermentation products could have been transferred with the inoculum. Thus, fresh media with only starter cultures of $R$. cohabitans or only dead biomass were incubated as control experiments. To investigate whether $R$. cohabitans can utilize putative carbon sources from the supernatant of the highly enriched Desulfobacterium culture, $30 \mathrm{~mL}$ centrifuged supernatant were filtered through $0.22 \mu \mathrm{m}$ pore-size membranes (Sarstedt, Germany), transferred to fresh serum bottles, and inoculated with $3 \mathrm{~mL}$ starter cultures of $R$. cohabitans. Non-inoculated bottles served as controls.

To further test whether $R$. cohabitans has the capability to ferment necromass, different substrates relevant to subsurface habitats were added to $50 \mathrm{~mL}$ bicarbonate-buffered freshwater medium and inoculated with $5 \mathrm{~mL}$ starter cultures of $R$. cohabitans. These substrates included $5 \mathrm{mM}$ sucrose, $5 \mathrm{mM}$ valine, $5 \mathrm{mM}$ pimelic acid, or $0.1 \%$ tryptone. Furthermore, living cells of the highly enriched Desulfobacterium culture grown in $75 \mathrm{~mL}$ cultures were harvested by centrifugation and added as substrate as described above. Fresh media with only starter cultures of $R$. cohabitans were incubated as carryover controls. 


\section{Gas chromatography}

Hydrogen was measured with a Shimadzu GC-8A Gas Chromatograph [17] or a Compact GC (Global Analyser Solutions, Breda, The Netherlands) [18]. For the former, high concentrations of hydrogen ( $>100$ parts per million by volume (ppmv)) were measured with a thermal conductivity detector, whereas low concentrations $(<100 \mathrm{ppmv})$ were measured with a reduction gas detector (RGD2 detector, Trace Analytical, Stanford, CA, USA). The measurements were calibrated with standard $\mathrm{H}_{2}$ gases (Messer Schweiz) (2 and $50 \mathrm{ppmv}$ for samples lower than $100 \mathrm{ppmv}, 1000 \mathrm{ppmv}$ for samples higher than $100 \mathrm{ppmv})$. For the latter, the GC was equipped with a Molsieve 5A pre-column and Porabond column $\left(\mathrm{CH}_{4}, \mathrm{O}_{2}, \mathrm{H}_{2}\right.$, and $\left.\mathrm{N}_{2}\right)$. The carrier gases were $\mathrm{N}_{2}$ for the $\mathrm{H}_{2}$ channel and $\mathrm{He}$ for the rest of the gases $\left(\mathrm{CH}_{4}, \mathrm{O}_{2}\right.$ and $\left.\mathrm{N}_{2}\right)$. The concentrations ( $\left.>100 \mathrm{ppmv}\right)$ were determined using a thermal conductivity detector.

\section{Sequencing and reconstruction of Spirochete genomes}

For comparative genomic analyses, three high-quality draft genomes were obtained, namely those of $R$. cohabitans, uncultured Spirochete bacterium bdmA 4, and uncultured Spirochete bacterium SA-8. The latter two genomes contained 16S rRNA gene sequences with $95 \%$ identity to $R$. cohabitans. The draft genome of the uncultured Spirochete bacterium SA-8 was derived from the metagenome of the terephthalate-degrading community TA, which is available on IMG/M (Integrated Microbial Genomes \& Microbiomes) as taxon object ID 3300001095 [6]. The draft genome of uncultured Spirochete bacterium bdmA 4 was reconstructed from the metagenome of another sulfate-reducing, naphthalenedegrading enrichment culture (designated Sob). Culture Sob was enriched from a creosote-contaminated wood preservation facility located in Soběslav, south of Bohemia (Czech Republic), as described by Kummel et al. [9]. Information on the shotgun sequencing, assembly, and binning processes [19] for the genome of $R$. cohabitans and the metagenome of enrichment culture Sob are described in the Supporting Information. The draft genomes of $R$. cohabitans, uncultured Spirochete bacterium bdmA 4, and uncultured Spirochete bacterium SA-8 were loaded into the MicroScope genome annotation platform and automatically annotated [20]. The annotations were manually curated and edited using the Magnifying Genome (MaGe) environment [21], and metabolic pathways were predicted using the integrated pathway tools of MaGe that are based on the KEGG (Kyoto Encyclopedia of Genes and Genomes) and MicroCyc databases [22, 23]. The presence of glycoside hydrolases and extracellular peptidases was evaluated using dbCAN $\left(\mathrm{E}<1 \mathrm{e}^{-10}\right.$ and cover fraction $\left.>0.4\right)[24]$ and MEROPS $\left(\mathrm{E}<1 \mathrm{e}^{-10}\right)[25]$, respectively.

\section{Phylogenetic analysis}

Potential genes encoding hydrogenase catalytic subunits were identified in the genomes of $R$. cohabitans and Desulfobacterium N47 by screening translated sequences through the web database HydDB [26]. Sequences were validated as hydrogenases if they encoded cysteinecontaining motifs characteristic of hydrogenases [27] and branched with hydrogenase reference sequences on phylogenetic trees. To infer evolutionary relationships, the source and reference sequences were aligned using the MUSCLE algorithm [28] and visualized on neighbor-joining phylogenetic trees [29] constructed with MEGA7 [30] and bootstrapped with 500 replicates. For genetic organization maps, protein/domain function was predicted using the CDD (Conserved Domain Database) [31] and iron-sulfur clusters were predicted by identifying conserved cysteine residues as previously described [27].

The phylogeny of the Spirochaetes was inferred using 16S rRNA gene sequences retrieved from the NCBI (National Center for Biotechnology Information) Genome Database. Sequences were aligned using the MUSCLE algorithm included in MEGA7 and trimmed to uniform length [30]. A maximum likelihood phylogenetic tree was constructed in MEGA7 using a general time reversible substitution model and uniform rates among sites. The tree was bootstrapped with 500 replicates.

\section{Mass spectrometry-based proteome analyses}

For proteomics, the enrichment culture N47 and the highly enriched Desulfobacterium culture (without $R$. cohabitans) were grown with naphthalene (four parallel cultures each). In addition, four cultures of $R$. cohabitans were grown with $10 \mathrm{mM}$ glucose and $0.1 \%$ yeast extract. The cultures were harvested after 6 weeks for the enrichment culture or 10 days for $R$. cohabitans and then immediately frozen at $-20^{\circ} \mathrm{C}$. The frozen cell pellets were molten at room temperature and taken up in $1 \mathrm{~mL}$ phosphate-buffered saline (3 $\mathrm{mM} \mathrm{Na}_{2} \mathrm{HPO}_{4}, 1.1 \mathrm{mM} \mathrm{KH} \mathrm{PO}_{4}$, and $155.2 \mathrm{mM} \mathrm{NaCl} ; \mathrm{pH}$ 7.4). The samples were lysed by ultrasonification in a Bioruptor $^{\circledR}$ (Diagenode) for $5 \mathrm{~min}$ to break the cells, release the proteome, and shear the genomic DNA (experimental settings: water bath temperature, $4{ }^{\circ} \mathrm{C}$; power setting, $\mathrm{H}$ position (High); sonication cycle, $30 \mathrm{sec}$ ON, $30 \mathrm{sec}$ OFF; total sonication time, $5 \mathrm{~min}$ ). The turbid solution was cleared by centrifugation $\left(12,000 \times \mathrm{g}, 4{ }^{\circ} \mathrm{C}, 5 \mathrm{~min}\right)$ and the supernatant was transferred to a fresh $1.5 \mathrm{~mL}$ Eppendorf tube. The protein concentration was determined by using a 


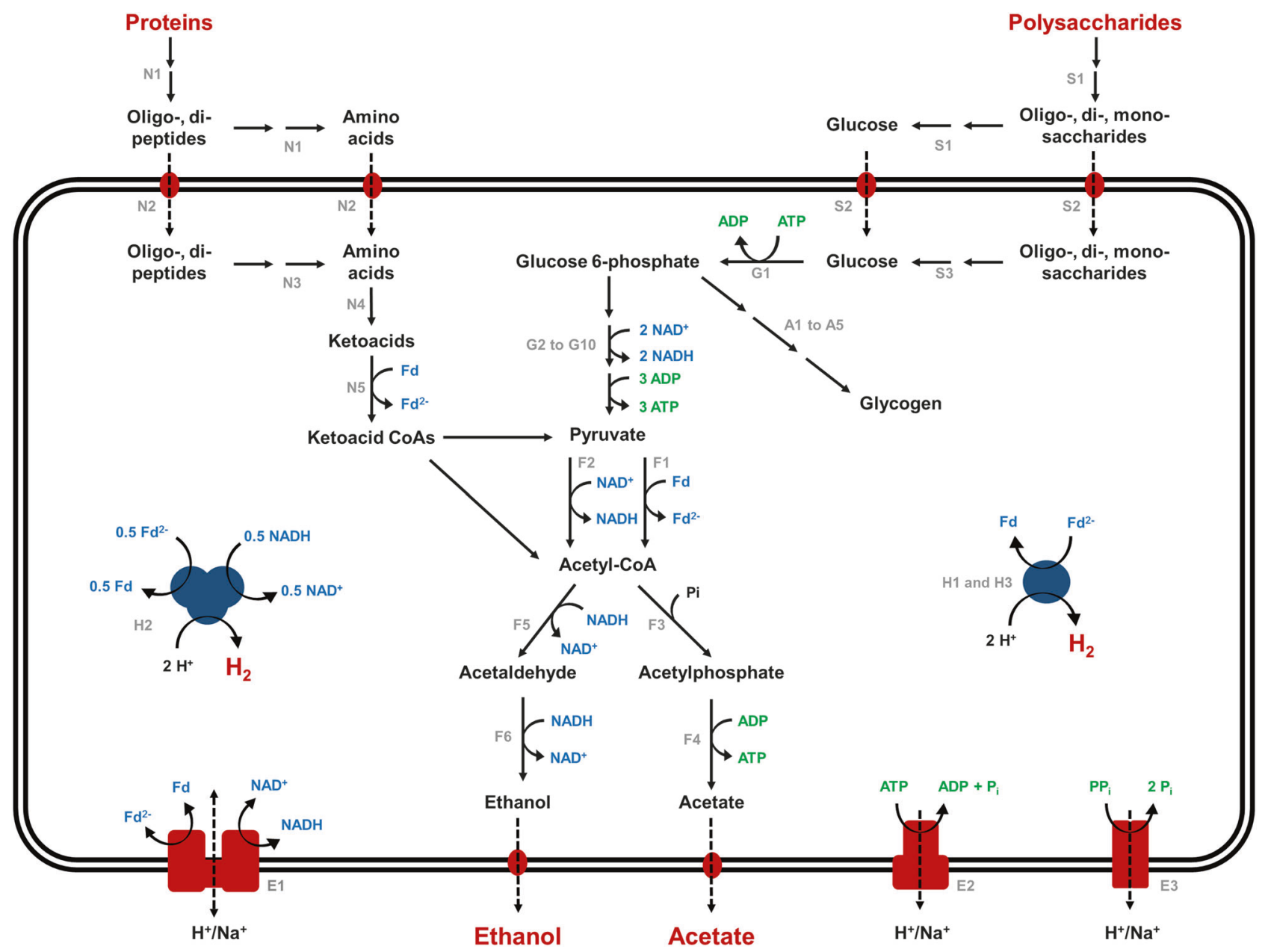

Fig. 1 Metabolic pathway reconstruction for Spirochaetes in hydrocarbon- and organohalide-contaminated environments. Genes for the illustrated pathways were detected in the genomes of $R$. cohabitans,

modified Bradford assay (RotiNanoquant; Roth) following the manufacturer's instructions. A detailed protocol for the subsequent sample preparation for Liquid chromatography tandem-mass spectrometry, as well as the settings during data acquisition and data analysis, can be found in the Supporting Information. The mass spectrometry proteomics data have been deposited to the ProteomeXchange Consortium via the PRIDE [32] partner repository (https://www.ebi.ac.uk/pride/archive/) with the dataset identifier PXD005624.

\section{Results}

\section{The genome of $R$. cohabitans HM suggests it is an obligate fermenter and hydrogen producer}

We sequenced and assembled a draft genome of $R$. cohabitans HM (2.82 Mb, estimated to be $98 \%$ complete, see Supporting Information) to develop hypotheses about its uncultured Spirochete bacterium bdmA 4, and uncultured Spirochete bacterium SA-8. The predicted functions of the putative proteins indicated by the numbers in the figure can be found in Table $\mathrm{S} 1$

ecophysiological role in the enrichment culture N47. Analysis of the metabolic capacity from the genome suggests that $R$. cohabitans performs a fermentative lifestyle. It is predicted to be capable of degrading proteins and carbohydrates, resulting in the production of the endproducts ethanol, acetate, and $\mathrm{H}_{2}$ (Fig. 1 and Supporting Information). In contrast, key genetic determinants of aerobic and anaerobic respiration, the tricarboxylic acid cycle, and carbon fixation via the reductive acetyl-CoA pathway are absent from the genome. This is consistent with previous substrate utilization tests [15].

Furthermore, we identified putative hydrogenase genes in the genomes of both $R$. cohabitans and Desulfobacterium N47. To gain insight into their potential ecophysiological roles, we classified them based on their amino acid sequence phylogeny and genetic organization according to a recently developed hydrogenase classification scheme [26, 27]. Seven putative [ $\mathrm{FeFe}]$-hydrogenase genes were identified in the genome of the Spirochete. Three are monomeric $[\mathrm{FeFe}]$ Group A1 or B hydrogenases (Fig. 2a, c), which are 
A

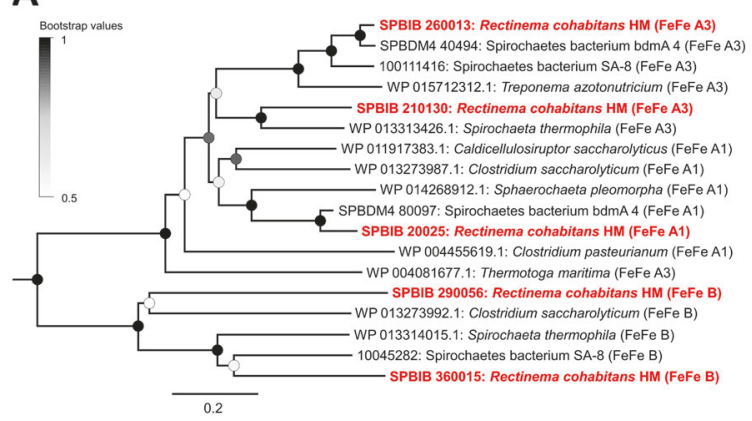

C

[FeFe] Group A1 / B hydrogenase operons (Rectinema cohabitans HM)

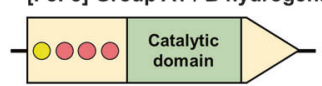

[FeFe] Group A3 hydrogenase operons (Rectinema cohabitans HM)

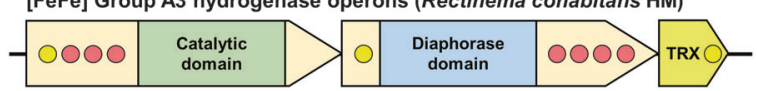

Fig. 2 Determinants of $\mathrm{H}_{2}$ metabolism in Spirochaetes in hydrocarbonand organohalide-contaminated environments. a Phylogenetic tree of the [FeFe]-hydrogenase catalytic subunit sequences detected in $R$. cohabitans, uncultured Spirochete bacterium bdmA 4, and uncultured Spirochete bacterium SA-8. b Phylogenetic tree of the [NiFe]-hydrogenase catalytic subunit sequences detected in Desulfobacterium N47. c Genetic organization of hydrogenases from $R$. cohabitans. d Genetic organization of the hydrogenase operon detected in Desulfobacterium N47. The illustrated tree for Figure $2 \mathrm{a}$ is condensed, with the full tree

known to couple oxidation of reduced ferredoxin to evolution of $\mathrm{H}_{2}$ during carbohydrate and protein fermentation in diverse bacteria [27, 33]. Two others encode trimeric electron-bifurcating [FeFe] Group A3 hydrogenases (Fig. 2a, c), which reversibly couple the oxidation of ferredoxin and $\mathrm{NADH}$ to the evolution of $\mathrm{H}_{2}$, thereby providing a novel mechanism to balance redox state and adenosine triphosphate demand [34]. The genome also encodes [FeFe] Group C1 and C3 hydrogenases (Figure $\mathrm{S} 1$ ), which are candidate subgroups involved in $\mathrm{H}_{2}$ sensing [26].

Re-analysis of the genome of the sulfate-reducing Desulfobacterium N47 [14] revealed the presence of two Group 1a and Group 1b [NiFe]-hydrogenases (Fig. 2b). These are known to couple $\mathrm{H}_{2}$ oxidation to cytochrome $c_{3}$ reduction in the respiratory chains of sulfate-reducing deltaproteobacteria. Whereas the former sequence is incomplete due to sequence coverage gaps, a complete operon encoding the $1 \mathrm{~b}$ enzyme and its cytochrome $c_{3}$ partner was detected (Fig. 2d) [27].

The genome-based predictions indicate that the association of the two predominant bacteria in the enrichment culture may be driven by the transfer of $\mathrm{H}_{2}$; we predict that $\mathrm{H}_{2}$ is produced during fermentation by $R$. cohabitans using [FeFe]-hydrogenases and subsequently consumed by the sulfate-reducing Desulfobacterium N47 using [NiFe]hydrogenases.
B



D

[NiFe] Group 1b hydrogenase operon (Desulfobacterium N47)

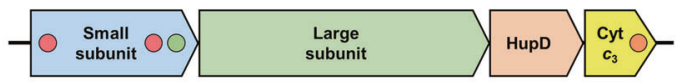

including all hydrogenases in Figure S1. The genetic organization of the Group 1a [NiFe] hydrogenase gene is not shown due to incomplete sequence coverage. Genetic organization diagrams are shown to scale and genes/domains are color-coded as follows: green, catalytic site; blue, secondary subunit; yellow, electron acceptor or donor; light orange, maturation factor. Redox-active centers are shown in circles, where yellow indicates $[2 \mathrm{Fe} 2 \mathrm{~S}]$ cluster, green $[3 \mathrm{Fe} 4 \mathrm{~S}]$ cluster, and red [4Fe4S] cluster

\section{Hydrogen is exchanged between $R$. cohabitans and Desulfobacterium N47}

To confirm that $R$. cohabitans is a fermentative $\mathrm{H}_{2}$ producer, we performed pure culture growth experiments with 10 $\mathrm{mM}$ glucose as the electron and carbon source. Gas chromatography demonstrated that $\mathrm{H}_{2}$ rapidly accumulated in the headspace during cultivation with glucose, resulting in $\mathrm{H}_{2}$ concentrations up to 400,000 ppmv after 2 weeks (Fig. 3a). Proteomic analysis of cells grown with glucose confirmed the expression of all hydrogenase-related genes with high peptide abundances (Table S2). Additionally, when grown with valine, sucrose, and tryptone, respectively, $R$. cohabitans produced almost threefold more hydrogen compared to controls after 1 week (Fig. 3b). This further confirms that $R$. cohabitans performs a proteolytic and saccharolytic lifestyle that results in hydrogen production.

The highly enriched Desulfobacterium culture was cultivated under sulfate-reducing conditions with naphthalene as carbon source. Meanwhile, the headspace was supplemented with a limited amount of $\mathrm{H}_{2}$ gas ( $\left.8285 \mathrm{ppmv}\right)$ as an additional electron donor for the reduction of sulfate (20 $\mathrm{mM})$. Consistent with genome-based predictions of hydrogenases for Desulfobacterium N47, we observed a gradual decrease in $\mathrm{H}_{2}$ concentration (Fig. 3a). Consumption of $\mathrm{H}_{2}$ by Desulfobacterium N47 was inhibited when $5 \mathrm{mM}$ 



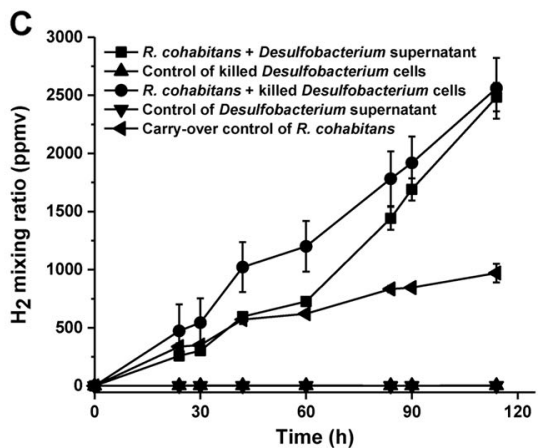

Fig. $3 \mathrm{H}_{2}$ oxidation and evolution under different growth conditions. a Interspecies hydrogen exchange between $R$. cohabitans and Desulfobacterium N47. Arrows indicate injection of sodium molybdate. b Hydrogen evolution with various substrates as carbon sources for $R$. cohabitans. $\mathbf{c}$ Hydrogen evolution with dead biomass and metabolites as carbon sources for $R$. cohabitans. Desulfobacterium in the figure represents biomass from the highly enriched Desulfobacterium culture as a substrate. Values are means of two or three individual incubations. Error bars indicate SD of biological replicates
Fig. 4 Quantitative proteomic analysis of the highly enriched Desulfobacterium culture (without $R$. cohabitans) and enrichment culture N47 grown in the presence of naphthalene. The volcano plot was generated using the PERSEUS software. The calculated $\log 2$ (fold change) ( $x$-axis) was then plotted against the corresponding $p$ values ( $y$-axis). Proteins to the left and above the significance line are significantly depleted in enrichment culture N47 and proteins to the right and above the significance line are significantly enriched in enrichment culture N47. The filled squares indicate proteins from $R$. cohabitans; the filled circles represent proteins from Desulfobacterium N47. The different colors highlight functional groups (for details see legend within figure)



sodium molybdate $\left(\mathrm{Na}_{2} \mathrm{MoO}_{4}\right)$, a potent inhibitor of sulfate reduction [35], was added at day 31 (Fig. 3a). This suggests that, in common with classical sulfate-reducing isolates [36], Desulfobacterium N47 directly couples $\mathrm{H}_{2}$ oxidation to sulfate reduction through an anaerobic respiratory chain.

Finally, in order to reveal a potential relationship between the two members, we monitored $\mathrm{H}_{2}$ concentrations in the enrichment culture N47 ( $R$. cohabitans + Desulfobacterium N47) with naphthalene and sulfate. The concentration of $\mathrm{H}_{2}$ remained constant ( $\left.2 \mathrm{ppmv}\right)$ for 1 month (Fig. 3a). However, a gradual increase in $\mathrm{H}_{2}$ concentration was observed when sulfate reduction by Desulfobacterium N47 was inhibited by the addition of sodium molybdate.
This suggests that there is a tight coupling between Spirochete-mediated fermentative $\mathrm{H}_{2}$ evolution and Deltaproteobacterium-mediated respiratory $\mathrm{H}_{2}$ consumption during the growth of Desulfobacterium with naphthalene as the sole supplemented carbon source and sulfate as the electron acceptor.

\section{Organic carbon derived from necromass fuels fermentation by $R$. cohabitans}

While the above results demonstrate that $\mathrm{H}_{2}$ is an important link between $R$. cohabitans and Desulfobacterium N47, the question remained about the origin of the reducing 
equivalents (i.e., electron sources) used for $\mathrm{H}_{2}$ production by $R$. cohabitans. Considering that $R$. cohabitans cannot utilize naphthalene and no other exogenous organic sources were added, the potential carbon sources for $R$. cohabitans include metabolites formed during naphthalene degradation and/or dead biomass from the deltaproteobacterium.

To address this question, we compared the proteomes of the highly enriched Desulfobacterium culture (without $R$. cohabitans, Figure S2) and the enrichment culture N47. The two proteomes revealed that only 5 out of 1266 proteins detected in total and assigned to Desulfobacterium N47 were differentially expressed between the two cultures, none of which could be assigned to a distinct ecophysiological function (Fig. 4). However, 53 proteins belonging to $R$. cohabitans were identified only in the enrichment culture N47 but not in the highly enriched Desulfobacterium culture (Table S3). Only a few proteins of $R$. cohabitans were detected in enrichment culture N47, most likely due to its relatively low abundance compared to the Desulfobacterium. Most of the R. cohabitans proteins expressed in the enrichment culture N47 were related to transporters for oligopeptides, dipeptides, amino acids, sugars, and dicarboxylic acids (Fig. 4 and Table S3). Other enriched enzymes include protease M41, glutamate dehydrogenase (amino acid catabolism), pyruvate-ferredoxin oxidoreductase (ferredoxin reduction), and components of the Sec translocon (secretion of hydrolases, insertion of transporters) (Table S3). This further supports that the Spirochaete performed fermentative degradation of polysaccharides and proteins in the enrichment culture N47.

As we did not add complex carbohydrates or peptides to the medium, these compounds are likely to originate from dead biomass of Desulfobacterium N47. We verified that $R$. cohabitans could use organic carbon sources derived from Desulfobacterium $\mathrm{N} 47$ to support fermentative $\mathrm{H}_{2}$ production by cultivating the Spirochaete with biomass of the highly enriched Desulfobacterium culture. Initially, the biomass was firstly autoclaved to produce artificial necromass and then added to culture bottles of $R$. cohabitans. After 3 days, the $\mathrm{H}_{2}$ production by $R$. cohabitans was twofold higher in fresh media incubations supplemented with either heat-killed cells or filtered supernatant of the highly enriched Desulfobacterium culture (Fig. 3c). Given that autoclaved biomass does not reflect the biomass present in the natural environment, we tested if biomass without any treatment could support growth of $R$. cohabitans. As Desulfobacterium N47 cannot survive under the defined growth conditions of $R$. cohabitans, it will die automatically and thus produce necromass due to lack of energy. After 7 days, the $\mathrm{H}_{2}$ production by $R$. cohabitans was 2.4 times higher compared to a control with inoculum only and no substrate (Fig. 3b). The two experiments show that $R$. cohabitans can use necromass and potentially exudates in the supernatant derived from Desulfobacterium N47 to support its obligately fermentative lifestyle.

Pimelate and other dicarboxylic acids are important downstream metabolites of anaerobic naphthalene degradation and have been detected in the supernatant of the enrichment culture N47 [10]. However, the genomic search did not support that $R$. cohabitans could utilize them as carbon sources, as we could not find genes for the $\beta$ oxidation of dicarboxylic acids [37]. Further substantiating this, substrate testing showed that pimelate does not stimulate hydrogen production (Fig. 3b). Together, the genome predictions (Fig. 1), substrate tests (Fig. 3), and proteomic data (Fig. 4) suggest that $R$. cohabitans primarily feeds on proteins and sugars derived from detrital biomass in the enrichment culture N47.

\section{Spirochaetes may generally mediate necromass recycling in anoxic hydrocarbon- and organohalide- contaminated habitats}

To further illuminate the ecological niche of Spirochaetes in polluted habitats, we compared the genome of $R$. cohabitans with the draft genomes of two uncultured environmental Spirochaetes that were reconstructed from metagenomics sequences: uncultured Spirochete bacterium SA-8, originating from a terephthalate-degrading, methaneproducing bioreactor [6], and uncultured Spirochete bacterium bdmA 4, derived from a naphthalene-degrading, sulfate-reducing culture enriched from a creosote spill [9].

Genome-based reconstructions suggest that, akin to $R$. cohabitans, both organisms are non-spiral Spirochaetes and obligate fermenters (Fig. 1 and Table S1). In addition, both genomes encode $[\mathrm{FeFe}]$ Groups $\mathrm{A}$ and $\mathrm{B}$ hydrogenases that are phylogenetically related to those of $R$. cohabitans (Fig. 2a and S1), suggesting that they also fermentatively produce $\mathrm{H}_{2}$, potentially sustaining associated hydrogenotrophic methanogens and sulfate reducers. Previously reported transcriptomic analysis supports that the uncultured Spirochete bacterium SA-8 participates in proteolytic amino acid degradation similar to $R$. cohabitans [6]. Revisiting the published genomes of the nonspiral Spirochaetes $S$. pleomorpha and $S$. globosa from trichloroethenedegrading cultures also indicated a potential for $\mathrm{H}_{2}$ production through saccharolytic mixed-acid fermentation (Fig. 2a and S1) [7]. In these cases, $\mathrm{H}_{2}$ probably serves as an electron donor to enhance reductive dechlorination activities of organohalide-respiring bacteria.

The 16S rRNA gene sequences of $R$. cohabitans, uncultured Spirochete bacterium bdmA 4, and uncultured Spirochete bacterium SA-8 are 95\% identical to each other, and form a common clade distinct from Treponema species in the 16S rRNA-based phylogenetic tree (Fig. 5 and S3). In the same clade, we could also identify numerous $16 \mathrm{~S}$ rRNA 


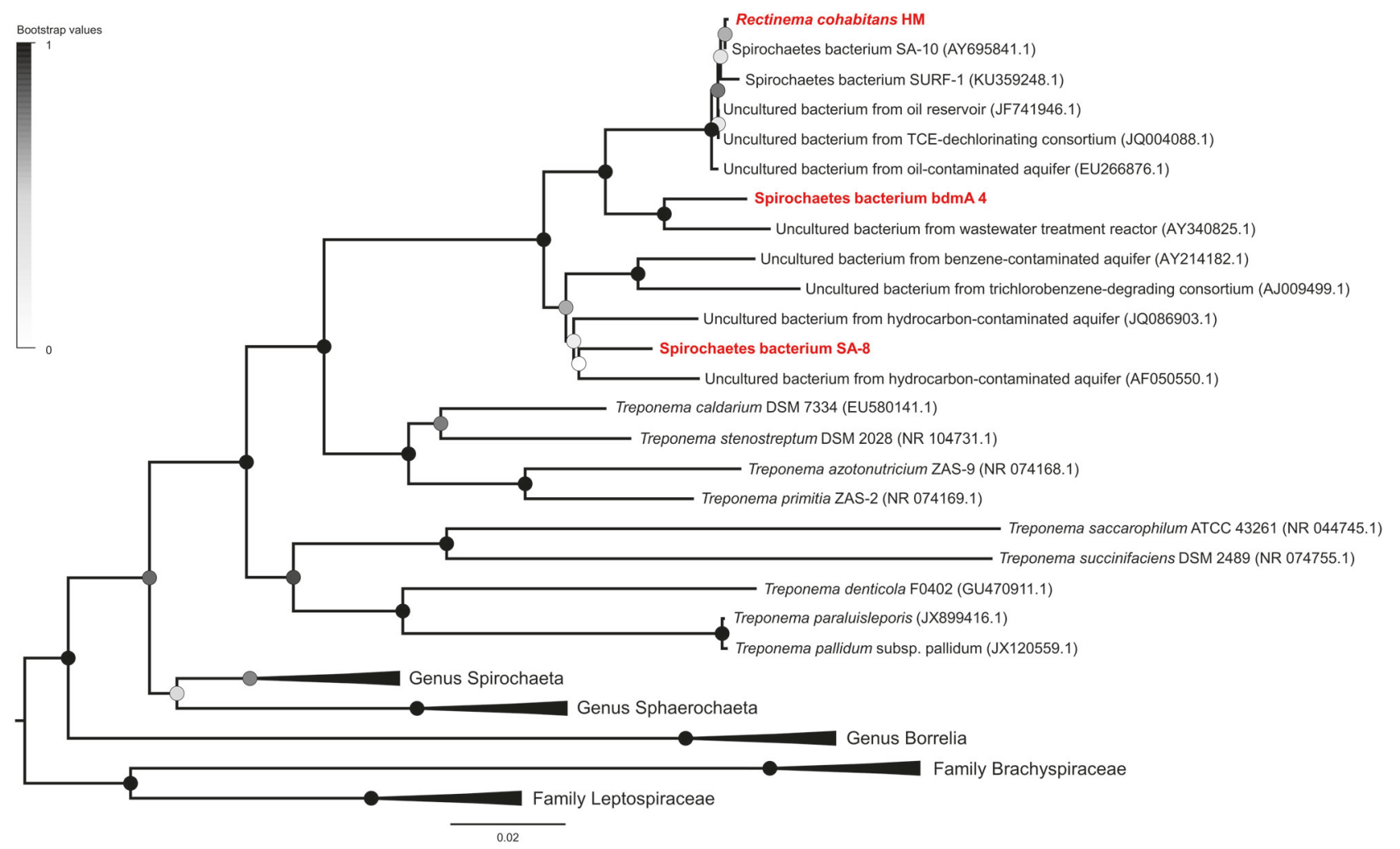

Fig. 5 Condensed maximum likelihood tree of partial 16S rRNA gene sequences of Spirochaetes showing the phylogenetic affiliation of $R$. cohabitans, uncultured Spirochete bacterium bdmA 4, and uncultured

gene sequences derived from contaminated aquifers or bioreactors containing hydrocarbons or organohalides as well as oil reservoirs. A small selection is shown in Figure 5. This indicates that Rectinema phylotypes are widely distributed in hydrocarbon- and organohalide-contaminated anoxic sites. The two strains $S$. globosa and S. pleomorpha are only distantly related to the three members of the genus Rectinema [15], suggesting that nonspiral morphology occurs in several lineages and that a similar metabolism is present in distinct groups of environmental Spirochaetes (Figure S3). Altogether, comparative genomic analyses suggest that these environmental nonspiral Spirochaetes fulfill ecophysiological functions similar to $R$. cohabitans in their communities: recycling necromass and providing hydrogen for hydrogenotrophic degraders of organohalides and hydrocarbons in these communities.

\section{Discussion}

Anaerobic degradation by indigenous microorganisms is the most important process that contributes to removal of anthropogenic organic pollutants in subsurface sites (e.g. contaminated groundwater) [38]. For some time, research on anaerobic degradation processes mostly focused on key
Spirochete bacterium SA-8. Phylogenetic trees were constructed by the maximum likelihood method and are bootstrapped with 500 replicates. The expanded tree is shown in Figure S3

players that are responsible for initial degradation of pollutants and/or reduction of electron acceptors (Fig. 6). However, it has become clear in recent years that the complete oxidation of organic pollutants to $\mathrm{CO}_{2}$ is based on labor sharing: participation of both key players for initial degradation of pollutants and secondary degraders for complete mineralization of catabolic by-products $[6,39$, 40]. Additionally, molecular surveys of anaerobic enrichment cultures or contaminated sites have revealed that many microbial species are not clearly correlated to degradation processes. They seem to be neither key players nor secondary degraders and are therefore unlikely to be involved in direct degradation of pollutants [6, 41]. Reflecting this, environmental Spirochaetes are common and abundant in contaminated sites and in enrichment cultures that degrade hydrocarbons and organohalides, but there are no indications that they are linked to degradation of either these compounds or by-products [15]. Our study of the enrichment culture N47 now provides experimental proof of one functional trait to these frequently detected organisms.

In the enrichment culture N47, the chemo-organoheterotrophic Desulfobacterium strain N47 degrades naphthalene as energy and carbon source with sulfate as electron acceptor, leading to primary biomass production in the absence of sunlight. Under nutrient-rich conditions, if there 


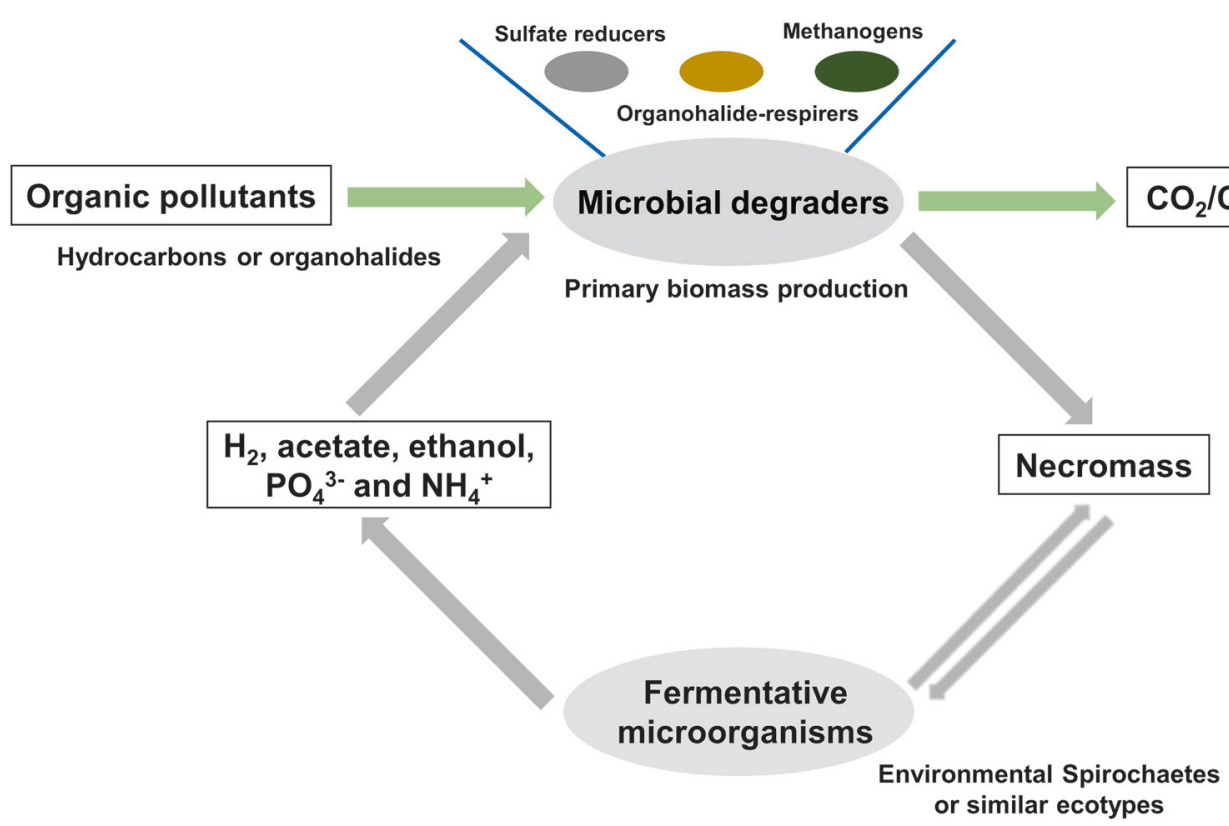

Fig. 6 A proposed conceptual model of a subsurface microbial loop for nutrient recycling at anoxic hydrocarbon- and organohalidecontaminated habitats. Hydrocarbons and organohalides serve as exogenous energy, electron, and carbon inputs, leading to primary biomass production in the absence of sunlight. Bacteria like environmental Spirochaetes use extracellular hydrolases and fermentative

is sufficient sulfate and naphthalene in the aqueous system (e.g., $>\sim 50 \mu \mathrm{M}$ ) [42], the sulfate reducer can live alone without the need of $R$. cohabitans to supply additional electron donors. In contrast, $R$. cohabitans relies on organic carbon sources derived from detrital biomass of Desulfobacterium N47 to sustain its growth. In turn, necromass degradation via hydrolysis and fermentation results in production of hydrogen and potentially short-chain fatty acids and alcohols. These products are then utilized by Desulfobacterium N47, thereby resulting in the recycling of necromass.

$R$. cohabitans is only one example out of many fermentative microorganisms that might mediate equivalent functions in necromass recycling. Even sulfate reducers themselves are quite versatile microorganisms and some perform hydrogenogenic fermentation when lacking electron acceptors such as sulfate or nitrate [43]. However, a fermenter that is more specialized for proteolysis and can secrete extracellular proteases and sacharolytic enzymes will most likely outcompete such sulfate reducers or other fermenters for necromass degradation. Consistently, the genomes of environmental Spirochaetes like $R$. cohabitans have genes encoding several extracellular peptidases (Merops family M23B, S8A, S26A, and S33) and carbohydrate-converting enzymes including hydrolases (Fig. 1 and Supporting Information). This is because necromass usually occurs in the form of biopolymers that pathways to recycle biomass. In turn, they release usable electron donors such as hydrogen and potentially nutrients like phosphorus and nitrogen to the system. This in turn may stimulate microbial degradation processes performed by key players, e.g., sulfate-reducing bacteria and organohalide-respiring bacteria, and other community members like methanogenic archaea

must be hydrolyzed outside the cell before metabolizing by microorganisms. Hence, exoenzymes play an essential role in initial processing by hydrolyzing complex organic matter derived from detrital compounds of other community members (e.g., sulfate reducers and fermenters) in the system [44]. Similar physiological properties are also well known for other organisms, such as Clostridia, that fulfill similar ecological roles in anoxic habitats.

In anoxic hydrocarbon- and organohalide-contaminated habitats, the biomass turnover rates are potentially high [45] because hydrocarbons (e.g., naphthalene and other aromatic compounds) or organohalides (e.g., trichloroethene) are usually present in relatively high concentrations in such habitats compared to pristine sites [38]. Their presence reshapes the abundances and interactions of indigenous microbes. In addition, they provide exogenous energy and carbon inputs, allowing for the buildup of significant biomass especially at contaminant plume fringes [38, 45]. For example, in the terephthalate-degrading bioreactor mentioned above, up to $10 \%$ of the terephthalate-derived carbon was converted to biomass which is a typical yield for anaerobic cultures [6]. In some benzene and toluenedegrading enrichment cultures, heterotrophic $\mathrm{CO}_{2}$ assimilation is also supposed to contribute greatly to biomass accumulation as evidenced from protein-based stable isotope probing (protein-SIP) and DNA-SIP experiments [41, 46]. Furthermore, at hydrocarbon- and organohalide- 
contaminated sites, high concentrations of organic solvents could challenge the integrity of cell membranes, inducing cell leakage or lysis [40, 47, 48].

Given the potential high biomass turnover, recycling of necromass is therefore important for ecosystem function and prevents loss of nutrients in contaminated ecosystems (Fig. 6). Most notably, it may contribute to enhancing biodegradation rates at contaminant plume fringes in groundwater or oil reservoirs where electron donors (e.g., hydrocarbons) and acceptors (e.g., sulfate or nitrate) meet in opposing gradients (Fig. 6) [38]. Firstly, during necromass recycling, the production of $\mathrm{H}_{2}$ and other substrates such as acetate and ethanol has frequently been reported to stimulate microbial blooms (the so-called "priming") [49] or serve as secondary substrates for co-metabolism, enhancing biodegradation rates [50]. Secondly, environmental Spirochaetes or similar ecotypes remineralize the necromass, which may also be important for recycling of nutrients such as nitrogen, phosphorus, and trace elements within a nutrient-poor subsurface ecosystem, increasing overall rates of biodegradation [51, 52].

At this point, we can only speculate on the importance of necromass recycling in the subsurface because quantitative data are not available. In our enrichment culture N47, $R$. cohabitans constituted $\sim 7 \%$ of the microbial population. Typical growth yields for anaerobic microorganisms are around $5-10 \%$ of the consumed substrates, as exemplified by the terephthalate-degrading culture mentioned above [6]. If necromass is the only electron and carbon source for $R$. cohabitans, at least a tenfold number of Desulfobacterium cells (i.e., $70 \%$ of total cell numbers) must have died to form necromass and serve as substrate for $R$. cohabitans to produce the observed $7 \%$ of the total cell numbers. Considering the actual proportion of $93 \%$ Desulfobacterium cells, this means that $163 \%$ of the total Desulfobacterium cell numbers (93\% living cells plus $70 \%$ necromass) must have grown. Thus, approximately $43 \%$ of all Desulfobacterium cells that have grown in the culture died and were recycled by $R$. cohabitans. The necessary difference in growth rate to account for the necromass recycling is quite small here. For example, if the culture grows exponentially from a $10 \%$ parent inoculum to full density (i.e., the cell numbers increased tenfold) in 6 weeks, the doubling time is approximately 13 days. Taken the described necromass recycling calculated above into consideration, it only shortens to $\sim 11$ days for the Desulfobacterium cells (i.e., the actual cell numbers increased 16.3-fold in 6 weeks). However, if such values of cell death and necromass recycling are transferable to subsurface habitats, and assuming that cell death is much higher under non-optimal conditions in the environment, we may speculate that fermentative organisms like $R$. cohabitans might constitute a subsurface microbial loop $[53,54]$ that can explain biomass recycling in the underground without a food chain (Fig. 6).

Acknowledgements We thank Masaru K. Nobu for supplying genome sequences of uncultured Spirochete SA-8, Melanie Klose for hydrogen measurement, Karine Labadie and Eric Mahieu for Illumina sequencing, and the Genoscope's LABGeM team for providing the MicroScope platform. We acknowledge partial funding from the FP7-KBBE MAGIC-PAH (project 245226) for (meta)genome sequencing. Xiyang Dong acknowledges a fellowship from the China Scholarship Council (File No. 201306060075). Chris Greening acknowledges funding from the ARC DECRA Fellowship (DE170100310) and ARC Discovery Project (DP180101762). Markus Kaiser acknowledges partial funding from the ERC starting grant No. 258413 and from the DFG grant No. INST 20876/127-1 FUGG. Rainer U. Meckenstock acknowledges funding from the ERC advanced grant EcOILogy (No. 666952).

\section{Compliance with ethical standards}

Conflict of interest The authors declare that they have no conflict of interest.

Open Access This article is licensed under a Creative Commons Attribution 4.0 International License, which permits use, sharing, adaptation, distribution and reproduction in any medium or format, as long as you give appropriate credit to the original author(s) and the source, provide a link to the Creative Commons license, and indicate if changes were made. The images or other third party material in this article are included in the article's Creative Commons license, unless indicated otherwise in a credit line to the material. If material is not included in the article's Creative Commons license and your intended use is not permitted by statutory regulation or exceeds the permitted use, you will need to obtain permission directly from the copyright holder. To view a copy of this license, visit http://creativecommons. org/licenses/by/4.0/.

\section{References}

1. Karami A, Sarshar M, Ranjbar R, Zanjani RS. The phylum Spirochaetaceae. In: Rosenberg E, DeLong EF, Lory S, Stackebrandt E, Thompson F, editors. The prokaryotes: other major lineages of bacteria and the archaea. Berlin, Heidelberg: Springer Berlin Heidelberg; 2014. p. 915-29.

2. Tan B, Fowler SJ, Abu Laban N, Dong X, Sensen CW, Foght J, et al. Comparative analysis of metagenomes from three methanogenic hydrocarbon-degrading enrichment cultures with 41 environmental samples. ISME J. 2015;9:2028-45.

3. Einsiedl F, Pilloni G, Ruth-Anneser B, Lueders T, Griebler C. Spatial distributions of sulphur species and sulphate-reducing bacteria provide insights into sulphur redox cycling and biodegradation hot-spots in a hydrocarbon-contaminated aquifer. Geochim Cosmochim Acta. 2015;156:207-21.

4. Müller S, Vogt C, Laube M, Harms H, Kleinsteuber S. Community dynamics within a bacterial consortium during growth on toluene under sulfate-reducing conditions. FEMS Microbiol Ecol. 2009;70:586-96.

5. Selesi D, Jehmlich N, von Bergen M, Schmidt F, Rattei T, Tischler $\mathrm{P}$, et al. Combined genomic and proteomic approaches identify gene clusters involved in anaerobic 2-methylnaphthalene degradation in the sulfate-reducing enrichment culture N47. J Bacteriol. 2010;192:295-306.

6. Nobu MK, Narihiro T, Rinke C, Kamagata Y, Tringe SG, Woyke T, et al. Microbial dark matter ecogenomics reveals complex 
synergistic networks in a methanogenic bioreactor. ISME J. 2015;9:1710-22.

7. Caro-Quintero A, Ritalahti KM, Cusick KD, Loffler FE, Konstantinidis KT. The chimeric genome of Sphaerochaeta: nonspiral spirochetes that break with the prevalent dogma in spirochete biology. MBio. 2012;3:pii: e00025-12

8. Ritalahti KM, Justicia-Leon SD, Cusick KD, Ramos-Hernandez N, Rubin M, Dornbush J, et al. Sphaerochaeta globosa gen. nov., sp. nov. and Sphaerochaeta pleomorpha sp. nov., free-living, spherical spirochaetes. Int J Syst Evol Microbiol. 2012;62:210-6.

9. Kummel S, Herbst FA, Bahr A, Duarte M, Pieper DH, Jehmlich N, et al. Anaerobic naphthalene degradation by sulfate-reducing Desulfobacteraceae from various anoxic aquifers. FEMS Microbiol Ecol. 2015;91:pii: fiv006

10. Meckenstock RU, Boll M, Mouttaki H, Koelschbach JS, Cunha Tarouco P, Weyrauch P, et al. Anaerobic degradation of benzene and polycyclic aromatic hydrocarbons. J Mol Microbiol Biotechnol. 2016;26:92-118.

11. Ontiveros-Valencia A, Tang Y, Krajmalnik-Brown R, Rittmann BE. Managing the interactions between sulfate- and perchloratereducing bacteria when using hydrogen-fed biofilms to treat a groundwater with a high perchlorate concentration. Water Res. 2014;55:215-24.

12. Duhamel M, Edwards EA. Microbial composition of chlorinated ethene-degrading cultures dominated by Dehalococcoides. FEMS Microbiol Ecol. 2006;58:538-49.

13. Meckenstock RU, Annweiler E, Michaelis W, Richnow HH, Schink B. Anaerobic naphthalene degradation by a sulfatereducing enrichment culture. Appl Environ Microbiol. 2000;66:2743-7.

14. Bergmann F, Selesi D, Weinmaier T, Tischler P, Rattei T, Meckenstock RU. Genomic insights into the metabolic potential of the polycyclic aromatic hydrocarbon degrading sulfatereducing Deltaproteobacterium N47. Environ Microbiol. 2011;13:1125-37.

15. Koelschbach JS, Mouttaki H, Pickl C, Heipieper HJ, Rachel R, Lawson PA, et al. Rectinema cohabitans gen. nov., sp. nov., a rodshaped spirochaete isolated from an anaerobic naphthalenedegrading enrichment culture. Int J Syst Evol Microbiol. 2017;67:1288-95.

16. Waldron LS, Ferrari BC, Gillings MR, Power ML. Terminal restriction fragment length polymorphism for identification of Cryptosporidium species in human feces. Appl Environ Microbiol. 2009;75:108-12.

17. Schuler S, Conrad R. Soils contain two different activities for oxidation of hydrogen. FEMS Microbiol Lett. 1990;73:77-83.

18. Guo K, Prévoteau A, Rabaey K. A novel tubular microbial electrolysis cell for high rate hydrogen production. J Power Sources. 2017;356:484-90.

19. Gkanogiannis A, Gazut S, Salanoubat M, Kanj S, Brüls T. A scalable assembly-free variable selection algorithm for biomarker discovery from metagenomes. BMC Bioinforma. 2016;17:311.

20. Vallenet D, Belda E, Calteau A, Cruveiller S, Engelen S, Lajus A, et al. MicroScope--an integrated microbial resource for the curation and comparative analysis of genomic and metabolic data. Nucleic Acids Res. 2013;41:D636-47.

21. Vallenet D, Labarre L, Rouy Z, Barbe V, Bocs S, Cruveiller S, et al. MaGe: a microbial genome annotation system supported by synteny results. Nucleic Acids Res. 2006;34:53-65.

22. Kanehisa M, Goto S. KEGG: Kyoto Encyclopedia of Genes and Genomes. Nucleic Acids Res. 2000;28:27-30.

23. Karpe PD, Latendresse M, Caspi R. The pathway tools pathway prediction algorithm. Stand Genom Sci. 2011;5:424-9.

24. Yin Y, Mao X, Yang J, Chen X, Mao F, Xu Y. dbCAN: a web resource for automated carbohydrate-active enzyme annotation. Nucleic Acids Res. 2012;40:W445-51.
25. Rawlings ND, Barrett AJ, Finn R. Twenty years of the MEROPS database of proteolytic enzymes, their substrates and inhibitors. Nucleic Acids Res. 2016;44:D343-50.

26. Søndergaard D, Pedersen CNS, Greening C. HydDB: a web tool for hydrogenase classification and analysis. Sci Rep. 2016;6:34212.

27. Greening C, Biswas A, Carere CR, Jackson CJ, Taylor MC, Stott $\mathrm{MB}$, et al. Genomic and metagenomic surveys of hydrogenase distribution indicate $\mathrm{H}_{2}$ is a widely utilised energy source for microbial growth and survival. ISME J. 2016;10:761-77.

28. Edgar RC. MUSCLE: multiple sequence alignment with high accuracy and high throughput. Nucleic Acids Res. 2004;32:1792-7.

29. Saitou N, Nei M. The neighbor-joining method: a new method for reconstructing phylogenetic trees. Mol Biol Evol. 1987;4:406-25.

30. Kumar S, Stecher G, Tamura K. MEGA7: Molecular Evolutionary Genetics Analysis Version 7.0 for Bigger Datasets. Mol Biol Evol. 2016;33:1870-4.

31. Marchler-Bauer A, Lu S, Anderson JB, Chitsaz F, Derbyshire MK, DeWeese-Scott C, et al. CDD: a Conserved Domain Database for the functional annotation of proteins. Nucleic Acids Res. 2011;39:D225-9.

32. Vizcaíno JA, Csordas A, del-Toro N, Dianes JA, Griss J, Lavidas I, et al. 2016 update of the PRIDE database and its related tools. Nucleic Acids Res. 2016;44:D447-56.

33. Wolf PG, Biswas A, Morales SE, Greening C, Gaskins HR. $\mathrm{H}_{2}$ metabolism is widespread and diverse among human colonic microbes. Gut Microbes. 2016;7:235-45.

34. Schuchmann K, Müller V. A bacterial electron-bifurcating hydrogenase. J Biol Chem. 2012;287:31165-71.

35. Banat IM, Lindström EB, Nedwell DB, Balba MT. Evidence for coexistence of two distinct functional groups of sulfate-reducing bacteria in salt marsh sediment. Appl Environ Microbiol. 1981;42:985-92.

36. Schwartz E, Fritsch J, Friedrich B. $\mathrm{H}_{2}$-metabolizing prokaryotes. In: Rosenberg E, DeLong EF, Lory S, Stackebrandt E, Thompson F, editors. The prokaryotes: prokaryotic physiology and biochemistry. Berlin, Heidelberg: Springer Berlin Heidelberg; 2013. p. 119-99.

37. Harrison FH, Harwood CS. The pimFABCDE operon from Rhodopseudomonas palustris mediates dicarboxylic acid degradation and participates in anaerobic benzoate degradation. Microbiology. 2005;151:727-36.

38. Meckenstock RU, Elsner M, Griebler C, Lueders T, Stumpp C, Aamand J, et al. Biodegradation: updating the concepts of control for microbial cleanup in contaminated aquifers. Environ Sci Technol. 2015;49:7073-81.

39. Kleinsteuber S, Schleinitz KM, Vogt C. Key players and team play: anaerobic microbial communities in hydrocarbon-contaminated aquifers. Appl Microbiol Biotechnol. 2012;94:851-73.

40. Lueders T. The ecology of anaerobic degraders of BTEX hydrocarbons in aquifers. FEMS Microbiol Ecol. 2017;93:pii: fiw220

41. Taubert M, Vogt C, Wubet T, Kleinsteuber S, Tarkka MT, Harms $\mathrm{H}$, et al. Protein-SIP enables time-resolved analysis of the carbon flux in a sulfate-reducing, benzene-degrading microbial consortium. ISME J. 2012;6:2291-301.

42. Morasch B, Annweiler E, Warthmann RJ, Meckenstock RU. The use of a solid adsorber resin for enrichment of bacteria with toxic substrates and to identify metabolites: degradation of naphthalene, $\mathrm{o}-$, and $\mathrm{m}$-xylene by sulfate-reducing bacteria. J Microbiol Methods. 2001;44:183-91.

43. Voordouw G. Carbon monoxide cycling by Desulfovibrio vulgaris Hildenborough. J Bacteriol. 2002;184:5903-11.

44. Christie-Oleza JA, Scanlan DJ, Armengaud J. "You produce while I clean up", a strategy revealed by exoproteomics during Synechococcus-Roseobacter interactions. Proteomics. 2015;15:3454-62. 
45. Griebler C, Lueders T. Microbial biodiversity in groundwater ecosystems. Freshw Biol. 2009;54:649-77.

46. Winderl C, Penning H, Netzer Fv, Meckenstock RU, Lueders T. DNA-SIP identifies sulfate-reducing Clostridia as important toluene degraders in tar-oil-contaminated aquifer sediment. ISME J. 2010;4:1314-25.

47. Rodriguez Martinez MF, Kelessidou N, Law Z, Gardiner J, Stephens G. Effect of solvents on obligately anaerobic bacteria. Anaerobe. 2008; 14:55-60.

48. Sherry A, Grant RJ, Aitken CM, Jones DM, Head IM, Gray ND. Volatile hydrocarbons inhibit methanogenic crude oil degradation. Front Microbiol. 2014;5:131.

49. Wrighton KC, Castelle CJ, Wilkins MJ, Hug LA, Sharon I, Thomas BC, et al. Metabolic interdependencies between phylogenetically novel fermenters and respiratory organisms in an unconfined aquifer. ISME J. 2014;8:1452-63.

50. Horvath RS. Microbial co-metabolism and the degradation of organic compounds in nature. Bacteriol Rev. 1972;36:146-55.

51. Head IM, Jones DM, Roling WFM. Marine microorganisms make a meal of oil. Nat Rev Micro. 2006;4:173-82.

52. Christie-Oleza JA, Sousoni D, Lloyd M, Armengaud J, Scanlan DJ. Nutrient recycling facilitates long-term stability of marine microbial phototroph-heterotroph interactions. Nat Microbiol. 2017;2:17100.

53. Kerner M, Hohenberg H, Ertl S, Reckermann M, Spitzy A. Selforganization of dissolved organic matter to micelle-like microparticles in river water. Nature. 2003;422:150-4.

54. Fenchel T. The microbial loop - 25 years later. J Exp Mar Biol Ecol. 2008;366:99-103. 